NASA-CR-189,599

NASA Contractor Report 189599

ICASE Report No. 92-2

NASA-CR-189599

19920009783

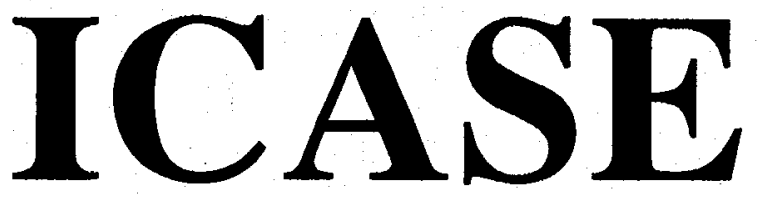

\title{
EFFECTIVE EQUATIONS AND THE INVERSE CASCADE THEORY FOR KOLMOGOROV FLOWS
}

Weinan E

Chi-Wang Shu

Contract No. NAS1-18605

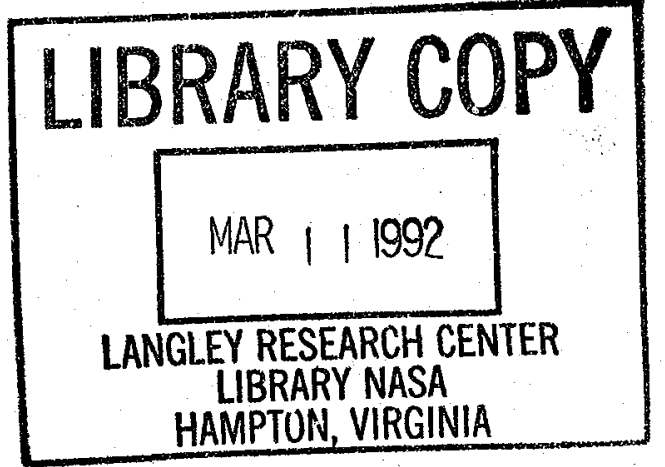

January 1992

Institute for Computer Applications in Science and Engineering NASA Langley Research Center

Hampton, Virginia 23665-5225

FOR REFERENCE

Operated by the Universities Space Research Association

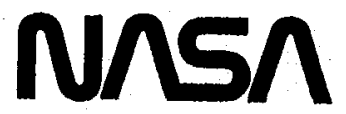

National Aeronautics and

Space Administration 



\title{
EFFECTIVE EQUATIONS AND THE INVERSE CASCADE THEORY FOR KOLMOGOROV FLOWS
}

\author{
Weinan $\mathrm{E}^{1}$ \\ School of Mathematics \\ Institute for Advanced Study \\ Princeton, NJ 08540 \\ Chi-Wang Shu ${ }^{2}$ \\ Division of Applied Mathematics \\ Brown University \\ Providence, RI 02912
}

\begin{abstract}
We study the two dimensional Kolmogorov flows in the limit as the forcing frequency goes to infinity. Direct numerical simulation indicates that in this limit the low frequency energy spectrum evolves to a universal $k^{-4}$ decay law. We derive effective equations governing the behavior of the large scale flow quantities. We then present numerical evidence that with smooth initial data, the solution to the effective equation develops a $k^{-4}$ type singularity at a finite time. This gives a convenient explanation for the $k^{-4}$ decay law exhibited by the original Kolmogorov flows.
\end{abstract}

\footnotetext{
${ }^{1}$ Research supported by ONR grant N00014-86-K-0691, ARO contract DAALO3-89-K-0039 and AFOSR90-0090.

${ }^{2}$ Research was supported by the National Aeronautics and Space Administration under NASA Contract No. NAS1-18605 while the author was in residence at the Institute for Computer Applications in Science and Engineering (ICASE), NASA Langley Research Center, Hampton, VA 23665.
} 



\section{$\S 1$. Introduction}

Toward the end of the 50's, Kolmogorov proposed to study the two-dimensional incompressible flow with a high frequency forcing term as a model to help understand the transition to turbulence and the inverse cascade process in a turbulent flow. These so-called Kolmogorov flows are described by the following equation:

$$
\left\{\begin{array}{l}
u_{t}+u \cdot \nabla u+\nabla p=\nu \Delta u+\nu k_{f}^{2}\left(\begin{array}{c}
\sin \left(k_{f} x_{2}\right) \\
0
\end{array}\right), \\
\nabla \cdot u=0 .
\end{array}\right.
$$

Here $\nu$ is the dynamic viscosity, $k_{f}$ is the forcing frequency. Since then, this problem has attracted a lot of attention. Considerable progress has been made on both the experimental and theoretical side. We refer to [10] for a discussion on the laboratory realization of the Kolmogorov flows. The theoretical side of this problem has mostly been concentrated on the hydrodynamic stability viewpoint. Linear stability of the basic Kolmogorov flow (a stationary shear flow proportional to the forcing) was studied in [6]. The amplitude equations for the weakly nonlinear theory were derived in $[9,15]$. It was found that this problem belongs to a wider class of problems which exhibit large scale instability. Later stage development of the Kolmogorov flow was extensively studied more recently in $[11,12]$ using a dynamical system viewpoint. Several scenarios of the transition to chaos and turbulence were identified by resorting to careful numerical simulations.

In this paper, we take a different approach and study Kolmogorov's problem in the following asymptotic limit:

$$
\nu=\frac{1}{k_{f}}=\varepsilon \rightarrow 0
$$

Our main interest is to understand the inverse cascade process in the Kolmogorov flow. Our extensive numerical results indicate that, even if we start with a smooth initial data with an exponentially decaying energy spectrum, the high frequency forcing term pumps energy into lower frequencies so that, beginning at a finite time, the energy spectrum exhibits a universal $k^{-4}$ algebraic decay law at the low frequencies. 
To explain this phenomenon, we derive the effective equations governing the evolution of the large scale flow quantities. We present strong numerical evidence that the solution of the effective equation with smooth initial data form a singularity at a finite time. Furthermore, the energy spectrum at the time of singularity formation is precisely $k^{-4}$.

We remark that in this paper the term "cascade" is used loosely to describe situations where either energy or enstrophy is shifted in the frequency space. We do not have evidence that energy or enstrophy is transferred continuously in the frequency space with nonzero flux at each wavenumber since we did not measure these quantities.

This paper is organized as follows. In the next section we present results of direct numerical simulation of the Kolmogorov flow in the limit as the forcing frequency goes to infinity. The emphasis will be placed on the evolution of the energy spectrum. In Section 3 , we derive the effecetive equations for the averaged quantities using multiple scale asymptotics. In Section 4, we present our numerical evidence for the singularity formation in the solutions of the effective equations. We conclude this paper in Section 5 by commenting on the relevance of our findings for more general flows, including three-dimensional flows.

\section{$\S 2$. The Inverse Cascade Phenomenon in the Kolmogorov Flows}

As we mentioned in the introduction, we are interested in studying the inverse cascade phenomena associated with the Kolmogorov flow in the following asymptotic limit:

$$
\nu=\frac{1}{k_{f}}=\varepsilon
$$

As can be easily seen from the derivations presented in the next section (see Remark 3.2), this is the only distinguished limit for which both the viscous force and the external high frequency force play important roles in the effective equations governing the evolution of large scale structures.

Under the scaling (2.1), equation (1.1) takes the following form:

$$
\left\{\begin{array}{l}
u_{t}^{\varepsilon}+u^{\varepsilon} \cdot \nabla u^{\varepsilon}+\nabla p^{\varepsilon}=\varepsilon \Delta u^{\varepsilon}+\frac{1}{\varepsilon}\left(\begin{array}{c}
\sin \frac{x_{2}}{\varepsilon} \\
0
\end{array}\right) \\
\nabla \cdot u^{\varepsilon}=0
\end{array}\right.
$$


To study the influence of the small-scale forcing on the evolution of the large scales, in particular, to study the evolution of the energy spectrum associated with the Kolmogorov flow, we have numerically integrated (2.2) using a number of initial velocity fields and studied the limit as $\varepsilon \rightarrow 0$. We discretize (2.2) using a Fourier-collocation method in space, and a third order Runge-Kutta method in time. More details of the numerical method will be given in Section 4. Our calculation differs from the previous calculations of Sulem et.al. [16] and Platt et.al [11] in that we studied as far as we can the limit as $\varepsilon \rightarrow 0$. In particular we computed the solutions of (2.2) with forcing wavenumbers $k_{f}=10,25,50,100$ (corresponds to $\varepsilon=0.1,0.04,0.02,0.01)$. In these cases the solutions exhibit a clear separation of scales for some time.

Here we report two examples of our computational results. We have experimented with other sets of initial data. In all cases we observe the same kind of phenomena as we report below.

In the first example we take the initial vorticity to be

$$
\omega_{0}=\frac{\partial u_{0}}{\partial x_{2}}-\frac{\partial v_{0}}{\partial x_{1}}=\sin ^{2} \frac{x_{1}}{2} \sin ^{2} \frac{x_{2}}{2}-\pi^{2}
$$

We computed the energy spectrum using the following formula:

$$
E(k)=\sum_{\left|\sqrt{k_{1}^{2}+k_{2}^{2}}-k\right|<\frac{1}{2}}\left(\left|\hat{u}\left(k_{1}, k_{2}\right)\right|^{2}+\left|\hat{v}\left(k_{1}, k_{2}\right)\right|^{2}\right)
$$

where $\hat{u}\left(k_{1}, k_{2}\right)$ is the $\left(k_{1}, k_{2}\right)$-th Fourier mode of $u$. Figure 1 is the log-log plot the energy spectrum at times $t_{j}=0.1,0.2, \cdots, 2.5$. Here $\varepsilon=0.04$, and the computation is done on a $400^{2}$ grid. It is clear that the energy spectrum consists of two parts: The high frequency part is dominated by the forcing frequency and its higher harmonics. The low frequency part corresponds to the large scale structure of the flow. Although it is not shown in Figure 1 , the energy spectrum at $t=0$ decays much faster than those plotted there. At early times the low frequency spectrum is very well separated from the high frequency part. At about $t=1$, the high end of the low frequency spectrum begins to merge with the low end of the high frequency spectrum. At the same time, the low end of the low frequency spectrum 
forms an envelope. This envelope is compared to a straight line with slope equal to -4 . They are close.

In the second example we choose a nonsmooth initial vorticity:

$$
\omega\left(x_{1}, x_{2}\right)=H\left(x_{1}\right) H\left(x_{2}\right)-\pi^{4}
$$

where $H(x)$ is the hat function on $[0,2 \pi]$ with height $\pi$. The other parameters are the same as in the first example. The energy spectrum for $t=0.1,0.2, \cdots, 1$ are plotted in Figure 2 . We observe basically the same phenomena as in Figure 1 except that here the initial data is much less smooth, therefore the low frequency spectrum settles down to an envelope much faster. The envelope is also compared to a straight line of slope -4 and is seen to be very close to that.

Although there exists a large literature on two-dimensional turbulence theory (for a review, see [2]), the discussions are much less sound than Kolmogorov's theory for threedimensional turbulence. We therefore seek our own explanation of this seemingly universal behavior of relaxation to a $k^{-4}$ law for the low frequency spectrum. In the next section, we will derive the effective equations governing the evolution of the large scale structures and therefore the low frequency part of the spectrum. In Section 4 we will study the effective equations numerically and present evidence that the energy spectrum for the solutions of the effective equations evolve to a $k^{-4}$ decay law, even with a smooth initial data. The time that this happens is close to the time when the envelope forms at the low frequency part of the spectrum of the Kolmogorov flows. Beyond this time the original Kolmogorov flows no longer exhibit separation of scales and the effective equations cease to be valid.

\section{$\S$ 3. Effective Equations for the Large Scales}

In this section, we derive effective equations governing the evolution of large scale flow quantities. Enormous efforts have been devoted to the the derivation of similar equations, the so-called "Reynolds equation", for general turbulent flows. The fact that turbulent flow has a continuum range of scales presents a serious difficulty to this objective. Our problem is drastically simplified since there is a separation of the scales which are active in the flow. 
As we pointed out in the last section, eventually separation of scales no longer holds and the flow exhibits a transition to a continuum of scales. This signals the breakdown of the effective equation, an issue which will be looked at more carefully in the next section.

We begin with the following proposition:

$$
\begin{gathered}
\left(\begin{array}{c}
u^{\varepsilon}(x, t) \\
v^{\varepsilon}(x, t)
\end{array}\right)=\left(\begin{array}{c}
\hat{u}(x, t) \\
\hat{v}(x, t)
\end{array}\right)+\left(\begin{array}{c}
w\left(x, t, \frac{x_{2}}{\varepsilon}\right) \\
z\left(x, t, \frac{x_{2}}{\varepsilon}\right)
\end{array}\right)+\varepsilon\left(\begin{array}{c}
u_{1}\left(x, t, \frac{x_{2}}{\varepsilon}\right) \\
v_{1}\left(x, t, \frac{x_{2}}{\varepsilon}\right)
\end{array}\right)+\cdots \\
p^{\varepsilon}(x, t)=\hat{p}(x, t)+\Pi\left(x, t, \frac{x_{2}}{\varepsilon}\right)+\varepsilon p_{1}\left(x, t, \frac{x_{2}}{\varepsilon}\right)+\cdots
\end{gathered}
$$

where $w\left(x, t, y_{2}\right), \Pi\left(x, t, y_{2}\right), u_{1}\left(x, t, y_{2}\right), p_{1}\left(x, t, y_{2}\right)$ etc. are periodic functions of $y_{2}$, and $<w(x, t, \cdot)>=0,\left\langle z(x, t, \cdot)>=0,<\Pi(x, t, \cdot)>=0\right.$ for every $x, t \in R^{2} \times R^{+}$. Substituting (3.1) into (2.2), and collecting equal orders of $\varepsilon$, we get a hierarchy of equations. The $O\left(\varepsilon^{-1}\right)$ equation are:

$$
\begin{gathered}
\nabla_{y} \cdot\left(\begin{array}{cc}
(\hat{u}+w)^{2} & (\hat{u}+w)(\hat{v}+z) \\
(\hat{u}+w)(\hat{v}+z) & (\hat{v}+w)^{2}
\end{array}\right)+\nabla_{y} \Pi=\Delta_{y}\left(\begin{array}{c}
w \\
z
\end{array}\right)+\left(\begin{array}{c}
\sin y_{2} \\
0
\end{array}\right), \\
\nabla_{y} \cdot(w, z)^{T}=0 .
\end{gathered}
$$

The solutions of (3.2) can be obtained explicitly:

$$
w=\frac{1}{\hat{v}^{2}+1}\left[\sin y_{2}-\hat{v} \cos y_{2}\right], \quad z=0, \quad \Pi=0
$$

We next come to the $O(1)$ equations:

$$
\begin{gathered}
\left(\begin{array}{c}
\hat{u}(x, t) \\
\hat{v}(x, t)
\end{array}\right)_{t}+\left(\begin{array}{c}
w \\
z
\end{array}\right)_{t}+\nabla_{x} \cdot\left(\begin{array}{cc}
(\hat{u}+w)^{2} & (\hat{u}+w)(\hat{v}+z) \\
(\hat{u}+w)(\hat{v}+z) & (\hat{v}+w)^{2}
\end{array}\right)+ \\
\nabla_{y} \cdot\left(\begin{array}{cc}
2(\hat{u}+w) u_{1} & (\hat{u}+w) v_{1}+(\hat{v}+z) u_{1} \\
(\hat{u}+w) v_{1}+(\hat{v}+z) u_{1} & 2(\hat{v}+z) v_{1}
\end{array}\right)+\nabla_{x} \hat{p}+\nabla_{x} \Pi+\nabla_{y} p_{1} \\
=2\left(\nabla_{x} \cdot \nabla_{y}\right)\left(\begin{array}{c}
w \\
z
\end{array}\right)+\Delta_{y}\left(\begin{array}{c}
u_{1} \\
v_{1}
\end{array}\right) \\
\nabla_{x} \cdot(\hat{u}+w, \hat{v}+z)^{T}+\nabla_{y} \cdot\left(u_{1}, v_{1}\right)^{T}=0 .
\end{gathered}
$$


Averaging both sides of (3.4) with respect to $y$, we get (written in conservative form):

$$
\left\{\begin{array}{l}
\left(\begin{array}{c}
\hat{u}(x, t) \\
\hat{v}(x, t)
\end{array}\right)_{t}+\nabla_{x} \cdot\left(\begin{array}{cc}
\hat{u}^{2} & \hat{u} \hat{v} \\
\hat{u} \hat{v} & \hat{v}^{2}
\end{array}\right)+\nabla_{x} \cdot\left(\begin{array}{cc}
<w^{2}> & <w z> \\
<w z> & <z^{2}>
\end{array}\right)+\nabla_{x} \hat{p}=0 \\
\nabla_{x} \cdot \hat{u}=0
\end{array}\right.
$$

Substituting in (3.5) the result of (3.3), we get

$$
\left\{\begin{array}{l}
\hat{u}_{t}+\hat{u} \hat{u}_{x_{1}}+\hat{v} \hat{u}_{x_{2}}+\left(\frac{1}{2\left(\hat{v}^{2}+1\right)}\right)_{x_{1}}+\hat{p}_{x_{1}}=0 \\
\hat{v}_{t}+\hat{u} \hat{v}_{x_{1}}+\hat{v} \hat{v}_{x_{2}}+\hat{p}_{x_{2}}=0 \\
\hat{u}_{x_{1}}+\hat{v}_{x_{2}}=0
\end{array}\right.
$$

This is the effective equation governing the evolution of the large scale quantities for the Kolmogorov flow. For the self-consistency of this asymptotic argument, we have to produce a candidate $\left(u_{1}, p_{1}\right)$ which solves the remainder of (3.4). To this end we write (3.4) as

$$
\begin{gathered}
\nabla_{y} \cdot\left(\begin{array}{cc}
2(\hat{u}+w) u_{1} & (\hat{u}+w) v_{1}+(\hat{v}+z) u_{1} \\
(\hat{u}+w) v_{1}+(\hat{v}+z) u_{1} & 2(\hat{v}+z) v_{1}
\end{array}\right)+\nabla_{y} p_{1}-\triangle_{y}\left(\begin{array}{c}
u_{1} \\
v_{1}
\end{array}\right)=f \\
\nabla_{y} \cdot\left(u_{1}, v_{1}\right)^{T}=-\nabla_{x} \cdot(\hat{u}+w, \hat{v}+z)^{T}=g
\end{gathered}
$$

where

$$
\begin{gathered}
-f=\left(\begin{array}{c}
-f_{1} \\
-f_{2}
\end{array}\right)=\left(\begin{array}{c}
\hat{u}(x, t) \\
\hat{v}(x, t)
\end{array}\right)_{t}+\left(\begin{array}{l}
w \\
z
\end{array}\right)_{t}+\nabla_{x} \cdot\left(\begin{array}{cc}
(\hat{u}+w)^{2} & (\hat{u}+w)(\hat{v}+z) \\
(\hat{u}+w)(\hat{v}+z) & (\hat{v}+w)^{2}
\end{array}\right) \\
+\nabla_{x} \hat{p}+\nabla_{x} \Pi-2\left(\nabla_{x} \cdot \nabla_{y}\right)\left(\begin{array}{c}
w \\
z
\end{array}\right) .
\end{gathered}
$$

In (3.7) and (3.8), $x$ and $t$ are viewed as parameters. Since $f$ and $g$ only depend on $y_{2}$, we seek a solution of (3.7) and (3.8) which depends only on $y_{2}$. The constructions above for $w, z, \Pi, \hat{u}, \hat{v}$ and $\hat{p}$ guarantee that the averages of $f$ and $g$ in $y_{2}$ over the period $[0,2 \pi]$ are zero. Therefore we solve $v_{1}$ from (3.8). The first equation of (3.7) will then give the solution $u_{1}$ whereas the second equation will give the solution $p_{1}$. It is easy to see that if we define

$$
\left(\begin{array}{c}
\tilde{u}^{\varepsilon}(x, t) \\
\tilde{v}^{\varepsilon}(x, t)
\end{array}\right)=\left(\begin{array}{c}
\hat{u}(x, t) \\
\hat{v}(x, t)
\end{array}\right)+\left(\begin{array}{c}
w\left(x, t, \frac{x_{2}}{\varepsilon}\right) \\
z\left(x, t, \frac{x_{2}}{\varepsilon}\right)
\end{array}\right)+\varepsilon\left(\begin{array}{c}
u_{1}\left(x, t, \frac{x_{2}}{\varepsilon}\right) \\
v_{1}\left(x, t, \frac{x_{2}}{\varepsilon}\right)
\end{array}\right)
$$




$$
\tilde{p}^{\varepsilon}(x, t)=\hat{p}(x, t)+\Pi\left(x, t, \frac{x_{2}}{\varepsilon}\right)+\varepsilon p_{1}\left(x, t, \frac{x_{2}}{\varepsilon}\right),
$$

then the equations (2.2) are satisfied by $\tilde{u}^{\varepsilon}, \tilde{v}^{\varepsilon}, \tilde{p}^{\varepsilon}$ with an error of order $\varepsilon$.

Remark 1: The difference between equation (3.6) and the usual 2-D Euler's equation is the appearance of the off-diagonal term in (3.6). This means that the linearized equation is weakly well-posed: high frequency perturbations will grow with a growth rate which is a polynomial in the wavenumber. This fact motivates us to look for finite time singularity formation in the solutions of (3.6) with smooth initial data.

Remark 2: If we change the forcing term in (1.1) to a different function, the form of the effective equation will not change. The off-diagonal term will usually change to a different function. In particular if the viscosity is scaled to vanish faster than $O(\varepsilon)$, then the added flux in the effective equation is singular in $\hat{v}$.

Remark 3: We notice that (3.6) conserves energy:

$$
\begin{aligned}
& \frac{1}{2} \frac{d}{d t} \int\left(\hat{u}^{2}+\hat{v}^{2}\right) d x_{1} d x_{2}=-\int \hat{u}\left(\frac{1}{2\left(\hat{v}^{2}+1\right)}\right)_{x_{1}} d x_{1} d x_{2} \\
= & \int \hat{u}_{x_{1}} \frac{1}{2\left(\hat{v}^{2}+1\right)} d x_{1} d x_{2}=-\int \hat{v}_{x_{2}} \frac{1}{2\left(\hat{v}^{2}+1\right)} d x_{1} d x_{2}=0 .
\end{aligned}
$$

\section{$\S$ 4. Singularity Formation in the Solutions of the Effective Equations}

In this section, we present numerical evidence which suggests that the solutions of (3.6) with analytic initial data develop singularities at finite time. Furthermore, at the time when singularity forms, the energy spectrum associated with the solutions decays like $k^{-4}$.

The numerical results presented below were computed using a Fourier-collocation method. We checked these results using both a second order centered differencing scheme and a fourth order ENO type scheme [14]. We remark that searching singularities numerically is a very subtle undertaking. It is important to check the numerical results using different methods since each method is likely to have its own numerical artifact when it comes to the computation of singularities.

Strictly speaking, demonstrating finite time singularity formation is an analytical problem. The ultimate answer has to come analytically, either by giving specific examples or 
by establishing a lower bound for the life span of the smooth solutions. Computers are limited to finite arithmetic and finite capacities. A numerical approach can at best give partial evidence, not the complete answer. Needless to say that we have tried the analytical approach and the problem seems to be very hard. For the moment, presenting numerical evidence is the best we can do to shed some light on our problem.

\section{$\S$ 4.1. Description of the Numerical Method}

For the spatial discretization, we used the Fourier-collocation method [1]. Roughly speaking, the differentiation operator is approximated in the Fourier space, whereas the nonlinear operations such as multiplication are done in the physical space. We use the intrinsic Cray FFT routines which considerably enhanced the performance of the code.

We observed that even for the computation of smooth solutions of the standard two dimensional incompressible Euler's equation, a certain amount of filtering is needed for the stability of the numerical method, and it is crucial to add the filters correctly. A robust way of adding the filters is to replace the Fourier multiplier $i k_{j}$ for the differentiation operator. $\frac{\partial}{\partial x_{j}}$ by $i k_{j} \varphi\left(\left|k_{j}\right|\right)$, where

$$
\varphi(k)=e^{-\alpha\left(\frac{k}{N}\right)^{m_{f}}}, \text { for }|k| \leq N
$$

Here $N$ is the numerical cutoff for the Fourier modes. $m_{f}$ is the order of the filter. $\alpha$ is chosen so that $\varphi(N)=e^{-\alpha}=$ machine accuracy. The machine accuracy on Cray-YMP with single precision is roughly $10^{-14}$. The accuracy of such an approximation scheme depends on the parameter $m_{f}$. For smooth functions $f(x)$, we have

$$
\left\|f^{\prime}(x)-D_{N} f(x)\right\|=O\left(N^{-m_{f}}\right)
$$

With $D_{N} f=F^{-1}(i k \varphi(|k|)) F f$ is the numerical approximation of $f^{\prime}(x)$. $F$ denotes the Fourier transform operator. Unless otherwise stated, the results presented in $\S 4.2$ were computed with $m_{f}=14$.

For the temporal discretization, we used the third order Runge-Kutta method designed in [14]. We used the third order version since it only requires three auxiliary arrays, whereas 
the fourth order version requires five auxiliary arrays. We take initial data that is periodic with period $D$ where $D=[0,2 \pi] \times[0,2 \pi]$.

\section{$\S$ 4.2. Numerical Results}

We present our numerical results for the case when the initial vorticity is the same as in the first example of Section 2: $\omega_{0}=\sin ^{2} \frac{x}{2} \sin ^{2} \frac{y}{2}-\pi^{2}$.

As a first hint for the singularity formation, we plotted the time history of the quantity $H_{1}(t)=\int_{D}|\nabla \omega|^{2} d x d y$. Figure 3 suggests that as $N \rightarrow+\infty, H_{1}(t)$ grows without bound, for $t>0.9$.

This information alone cannot be used as a solid evidence for singularity formation, since it only says that the quantity $H_{1}(t)$ is poorly resolved for $t>0.9$. Next we plotted in Figure 4 the time evolution of the energy spectrum (on a log-log scale) associated with the numerical solutions obtained with three different resolutions: $128^{2}, 256^{2}$ and $400^{2}$. We observe that at $t=1$, a bump develops near the cutoff frequency on the energy spectrum. This indicates that at this time, the numerical solutions are not very well resolved. We also observe that the energy spectrum at $t=0.9$ is well represented by the numerical solutions obtained on the $128^{2}$ and $256^{2}$ grids, and it fits very well with a straight line with slope -4. This is a stronger indication that at this time, the energy spectrum of the solution no longer decays exponentially, but decays algebraically as $k^{-4}$.

To determine more precisely when algebraic decay sets in, we use the techniques proposed by Sulem, Sulem and Frisch [17]. Let $f(z)$ be a function which is analytic in the strip $|\operatorname{Im} z|<\alpha$, and has a branch-cut singularity $z^{\beta}$ on $|\operatorname{Im} z|=\alpha$, then its Fourier transform $\hat{f}(k)$ decays like

$$
\hat{f}(k) \sim C k^{-\beta} e^{-\alpha k}, \quad|k| \rightarrow+\infty .
$$

Imagine the following scenario for the singularity formation in the solutions of (3.6). Instead of solving (3.6) in the real space $R^{2}$, we solve (3.6) in the complex space $\left(z_{1}, z_{2}\right) \in C^{2}$. Initially the solution is an entire function. At later time the solution develops branch point 
of order $\beta(t)$ at $z(t)$ with $|\operatorname{Im} z(t)|=\alpha(t)>0$, but it is analytic in the strip $|\operatorname{Im} z|<\alpha(t)$. If this branch point travels to the real axis at a finite time $t^{*}$, then the solution of (3.6), solved in the real space, develops a singularity at $t=t^{*}$.

This scenario has been proposed for a number of problem in fluid mechanics, notably the formation of singularity in the evolution of a vortex sheet. (see $[8,3,13]$ ). In this case, there are strong numerical as well as analytical evidence which confirms this picture.

To check whether this provides a plausible picture for our problem, we checked numerically the validity of proposition 4.3 for the energy spectrum. To do that we choose three consecutive wave numbers $(k-1, k, k+1)$, and solve

$$
E(\tilde{k}, t)=C \tilde{k}^{-\beta} e^{-\alpha \tilde{k}}, \quad \text { for } \quad \tilde{k}=k-1, k, k+1,
$$

with double precision arithmetic (on Cray-YMP). At each fixed time, we get three functions $C(k, t), \beta(k, t), \alpha(k, t)$. These functions depend on $k$. In order that (4.3) be a good estimate for the energy spectrum of the solutions of (3.6), asymptotically as $k \rightarrow \infty$, the functions $C(k, t), \beta(k, t)$ and $\alpha(k, t)$ should not depend on $k$ in this limit. In Figure 5 we plotted the functions $\alpha(k, t)$ from $t=0.4$ to $t=1$ with constant time increments. The computation is done on a $256^{2}$ grid. It is clear from Figure 5 that for fixed $t, \alpha(k, t)$ is almost a constant for $20<k<60$. For $k<20, \alpha(k, t)$ exhibits fluctuations since $k$ is not yet in the asymptotic regime. For $80<k<128$, the energy density is at the order of $10^{-32}$ which is below machine accuracy even if double precision is used. These wavenumbers are needed only to enforce smooth decay for the Fourier coefficients down to the machine accuracy. Figure 6 displays similar results for a computation using a $400^{2}$ grid. The fluctuations at high wavenumber is a manifestation of trying to fit machine zeros with proposition 4.3. The functions $C(k, t)$ and $\beta(k, t)$ exhibit the same feature.

Now we can average the values of $\alpha$ over the intermediate wave numbers and safely regard the averaged value (as a function of $t$ ) as the width of the analyticity strip of the solution at that time. This function will be denoted by $\bar{\alpha}(t)$. The corresponding function for $\beta$ will be denoted by $\bar{\beta}(t)$. Displayed in Figure 7 is the function $\bar{\alpha}(t)$ for two different numerical resolutions: $256^{2}$ and $400^{2}$. Results obtained on both grids indicate that the 
width of the analyticity strip for the solution vanishes at $t^{*} \approx 0.9$, suggesting a singularity formation at this time. The corresponding results for $\bar{\beta}$ are displayed in Figure 8 . As $t \rightarrow t^{*}$, $\bar{\beta}(t)$ converges to 4 .

The other numerical parameter in our method is the order of the filter. Figures 9 and 10 display the results for $\bar{\alpha}, \bar{\beta}$ as we vary the order of the filter. We see that the approximated values of $\alpha, \beta$ are not sensitive to the change of the filters. However when the filter is too weak $\left(m_{f}=15\right)$, the numerical solution deteriorates drastically at a time before $t^{*}$, since it can no longer handle the large gradients developed in the vorticity field. The computed value fluctuates much more, therefore the result is not shown in Figure 10. This is not unexpected since in general $\beta$ is much more sensitive than $\alpha$.

To get an idea about the nature of the singularity, we display in Figures 11 and 12 the time evolution of $u$ and $\omega$ at $y=\frac{2 \pi}{3}$ with $t=0.3,0.6,0.9$. At $t=0.9$, a cusp seems to have formed on the profile of $\omega$. Contour plots of $\omega$ show that these cuspy structures occur on isolated lines.

The behavior of the solutions reported above seems to be generic. It occurs in other calculations we did using different initial data.

\section{§5. Concluding Remarks}

As we mentioned in the introduction, the Kolmogorov flow belongs to a large class of problems which exhibit large scale instability. A typical example in three dimension is the $\mathrm{ABC}$ flow which is known to be unstable to large scale perturbations [7]. In principle the ideas and methods presented above should apply to these problems also, although to actually carry out the program is difficult mainly because the computational cost increases drastically for three-dimensional problems. However, it is clear that such instabilities will trigger the transport of energy from small scales to large scales, thereby effecting the mean flow quantities.

One can also attempt to study the interaction of different scales by studying the solutions with two-scale initial data. Formally one can use multiple scale asymptotics to derive 
effective equations for the large scales. This is done in $[5,4]$. However, a closer look at these effective equations reveals that the linearized equations are exponentially ill-posed: high frequency perturbations grow at a rate that is exponential in the wavenumber. The reason for this is very simple. The incompressibility condition forces the microstructure to be shear flows. In the limit of infinite scale separation, the microstructure appears like stacks of vortex sheets when viewed at macro-scales. The linear ill-posedness is simply a manifestation of the Kelvin-Helmholtz instability.

In such situations multiple scale asymptotics does not provide useful information since it is based on the hypothesis of scale separation. For the Kolmogorov flow, we are saved since there is a fixed time interval in which the scales are separated and we can get useful information from two-scale expansions. As one might expect, beyond the critical time $t^{*}$, the two scales in the Kolmogorov flow merge and the effective equation cease to be valid. This is checked numerically with the following procedure. The effective equation (3.6) is integrated numerically using a finite difference method with a built-in nonlinear numerical viscosity to avoid oscillations coming from large vorticity gradient [14]. The energy spectrum of the numerical solution is found to be decaying increasingly slower. This differs sharply from the energy spectrum of the original Kolmogorov flow since the latter approaches an asymptote after the critical time.

Acknowledgement: The numerical computation reported here was done on the CRAY-YMP computers at the Pittsburgh Supercomputing Center. This work was done while the first author was a visiting member at the Courant Institute of Mathematical Sciences under the support of ARO contract DAAL03-89-K-0039 and AFOSR-90-0090. The second author was supported by ARO contract DAAL03-91-G-0123, NASA NAG1-1145, and NAS1-18605 while in residence at ICASE. 


\section{References}

[1] C. Canuto, M. Y. Hussaini, A. Quarteroni and T. A. Zang, Spectral Methods in Fluid Dynamics, Springer-Verlag, New York, 1987.

[2] R. H. Kraichnan and D. Montgomery, Two-dimensional turbulence, Rep. Prog. Phys. 43,547 (1980).

[3] R. Krasny, A study of singularity formation in a vortex sheet by the point-vortex approximation, J. Fluid Mech., 167, 65, 1986.

[4] V. P. Maslov, Coherent structures, resonances, and asymptotic non-uniqueness for Navier-Stokes equations with large Reynolds numbers, Russ. Math. Survey, 41:6 (1986), $23-42$.

[5] D. Mclaughlin, G. Papanicolaou and O. Pironneau, Convection of microstructures and related problems, SIAM J. Appl. Math. 45, 780-807 (1985).

[6] L. D. Meshalkin, and Ya. G. Sinai, Investigation of the stability of a stationary solution of a system of equations for the plane movement of an incompressible viscous liquid, J. Appl. Math. (PMM), 25, 1140-1143, (1961).

[7] H. K. Moffatt, Magnetostatic equilibria and analogous Euler flows of arbitrarily complex topology. Part 2. Stability considerations, J. Fluid Mech., 166, 359-378 (1986).

[8] D. W. Moore, The spontaneous appearance of a singularity in the shape of an evolving vortex sheet, Proc. R. Soc. Lond. A 365, 105, 1979.

[9] A. A. Nepomnyachtchyi, Prikl. Math. Makh. 40 (5), 886(1976).

[10] A. M. Obukhov, Russ. Math. Survey, 38, 113 (1983).

[11] N. Platt, L. Sirovich and N. Fitzmaurice, An investigation of chaotic Kolmogorov flows, ICASE Report No. 90-50, 1990. 
[12] Z. S. She, Instabilites et dynamique a grande echelle en turbulence, Thesis, Universite de Paris VII, Sept. 1987.

[13] M. Shelley, A study of singularity formation in vortex sheet motion by a spectrally accurate vortex method, J. Fluid Mech., to appear.

[14] C. W. Shu, and S. Osher, Efficient implementation of essentially non-oscillatory shockcapturing schemes, II, J. Comp. Phys., 83, 32-78 (1989).

[15] G. I. Sivashingsky, Weak turbulence in periodic flows, Physica D 17, 243-255, (1985).

[16] P. L. Sulem, Z. S. She, H. Scholl, and U. Frisch, Generation of large-scale structures in three-dimensional flow lacking parity-invariance, J. Fluid Mech., vol. 205, pp. 341-358 (1989).

[17] C. Sulem, P. Sulem and U. Frisch, Tracing complex singularities with spectral methods, J. Comp. Phys. 50, 138, 1983. 


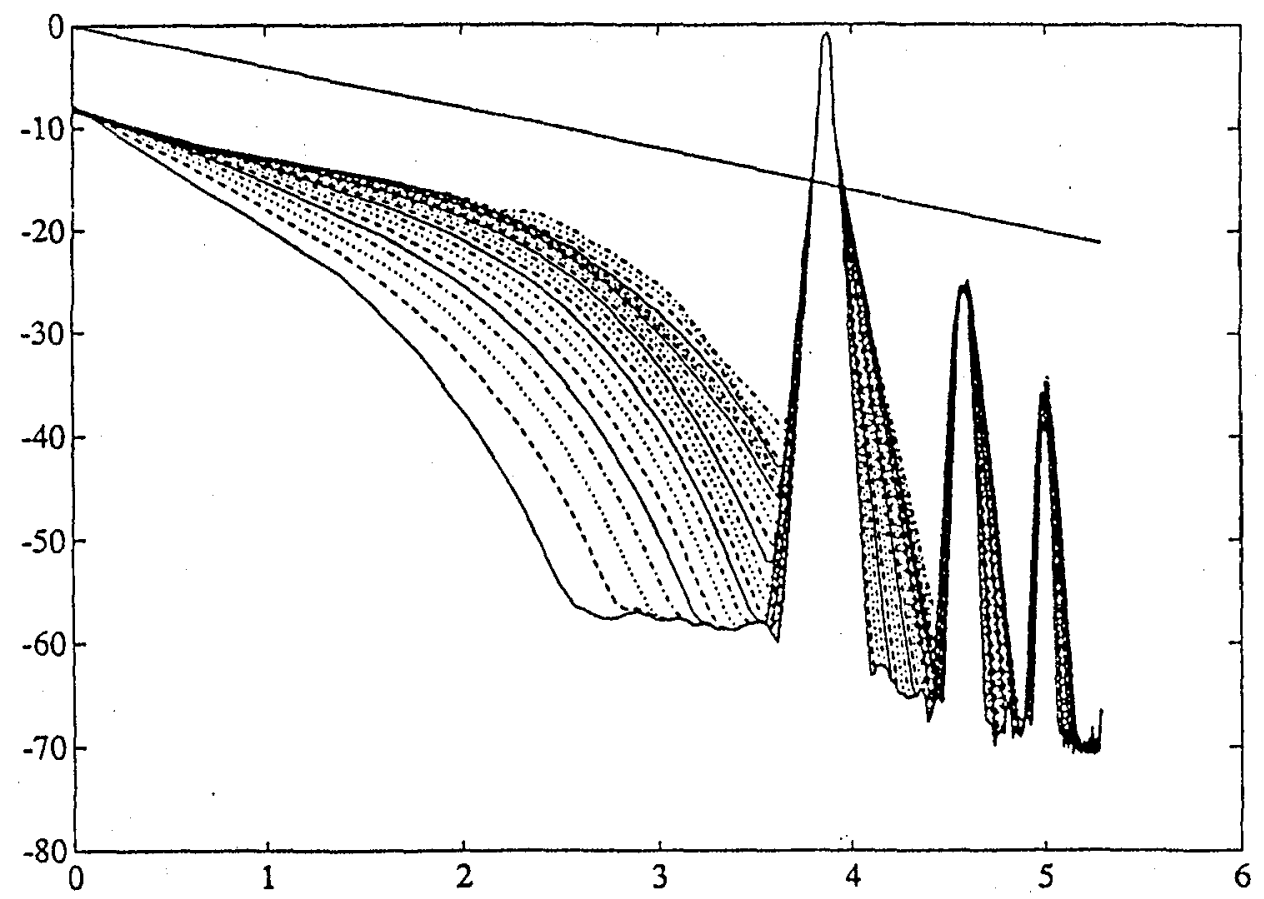

Figure 1. Log-log plot of the time evolution of the energy spectrum with initial data (2.3) at $t=0.1,0.2, \cdots, 2.5 . \varepsilon=0.04$. $t$ increases upward. The energy spectrum is compared to a line of slope -4 .

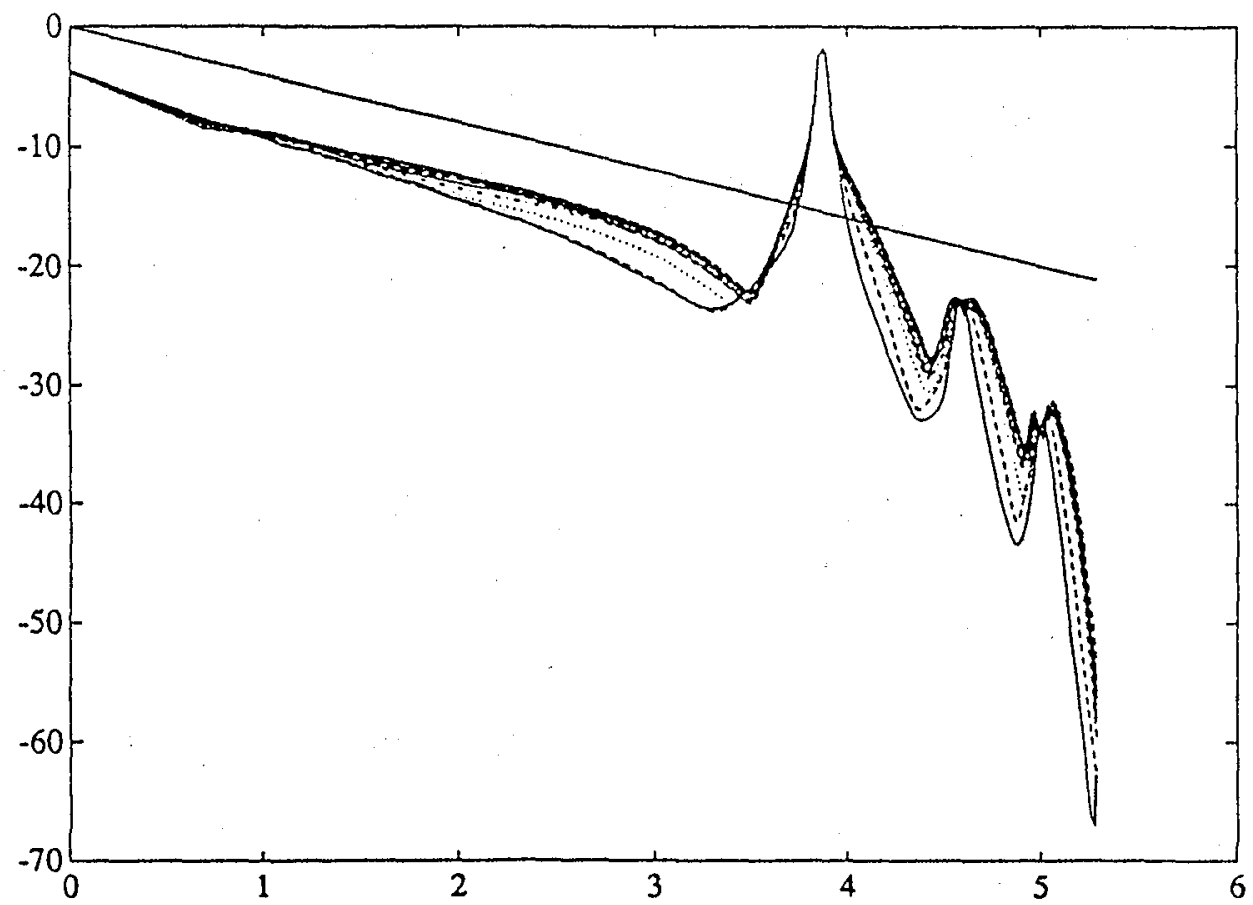

Figure 2. Log-log plot of the time evolution of the energy spectrum with initial data (2.5) at $t=0.1,0.2, \cdots, 1 . \varepsilon=0.04 . t$ increases upward. The energy spectrum is compared to a line of slope -4 . 


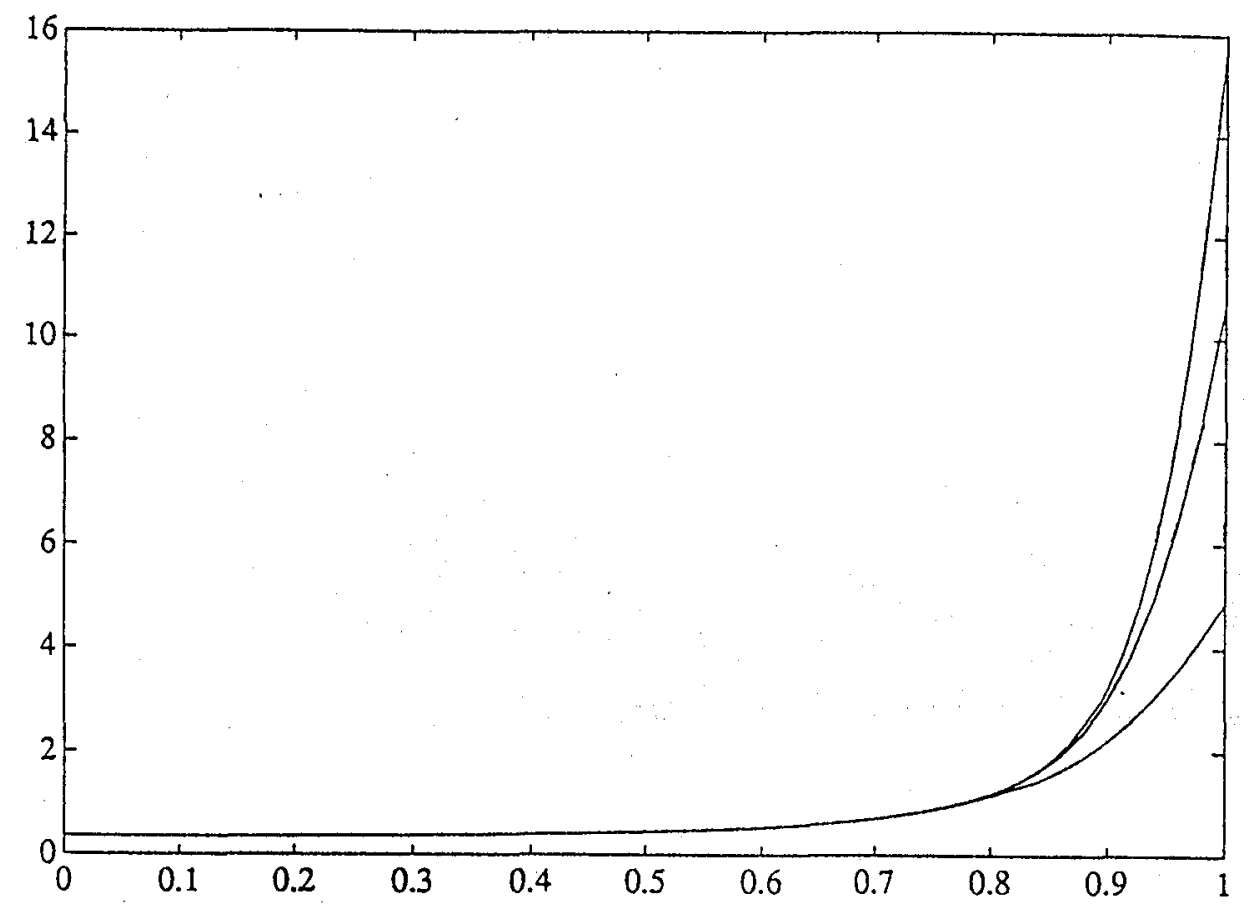

Figure 3. From bottom to top, time history of $H_{1}(t)$ on computational grids: $128^{2}, 256^{2}, 400^{2}$.

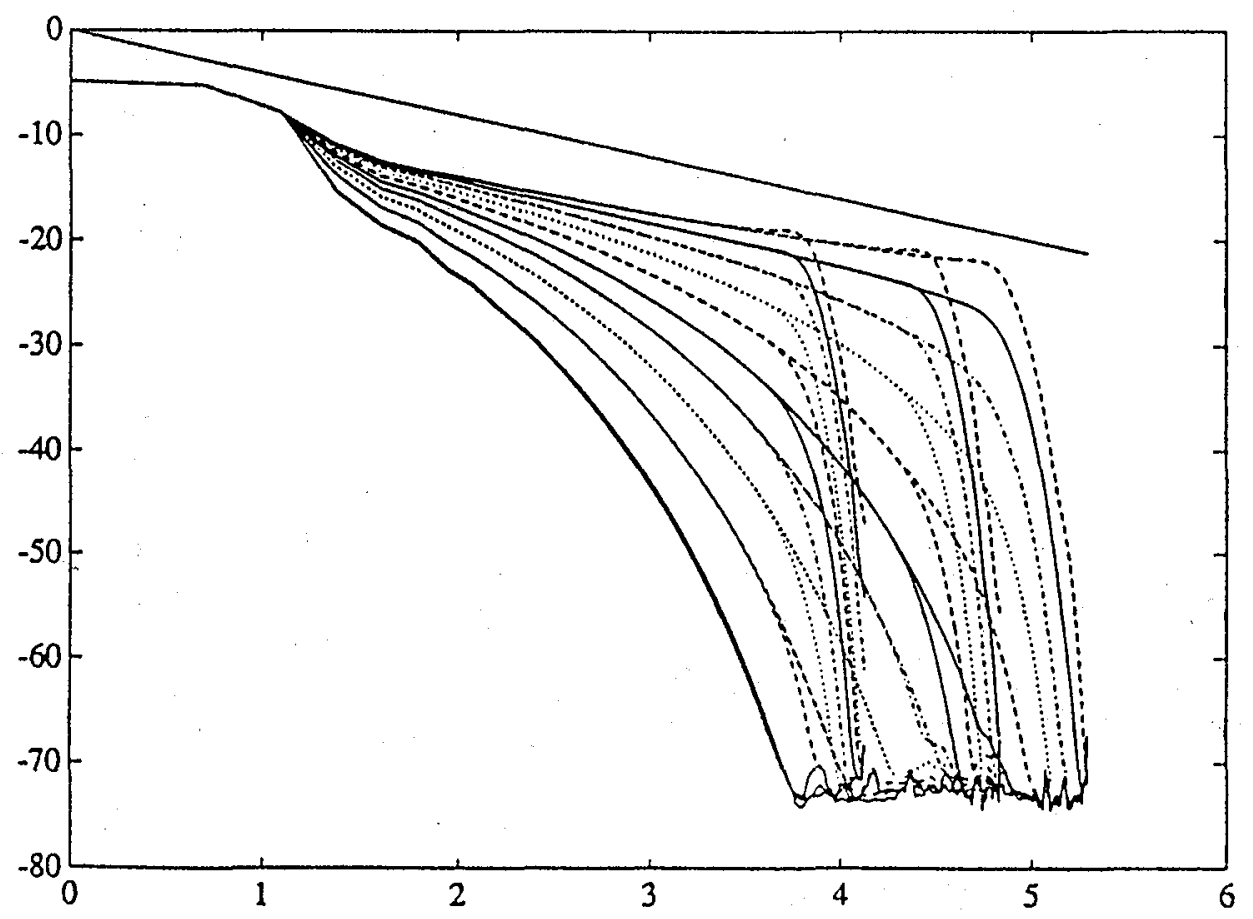

Figure 4. Log-log plot of the time evolution of the energy spectrum computed on grids: $128^{2}, 256^{2}$ and $400^{2}$. From bottom to top: $t=0.1,0.2, \cdots, 1$.The energy spectrum is compared to a line of slope -4 . 


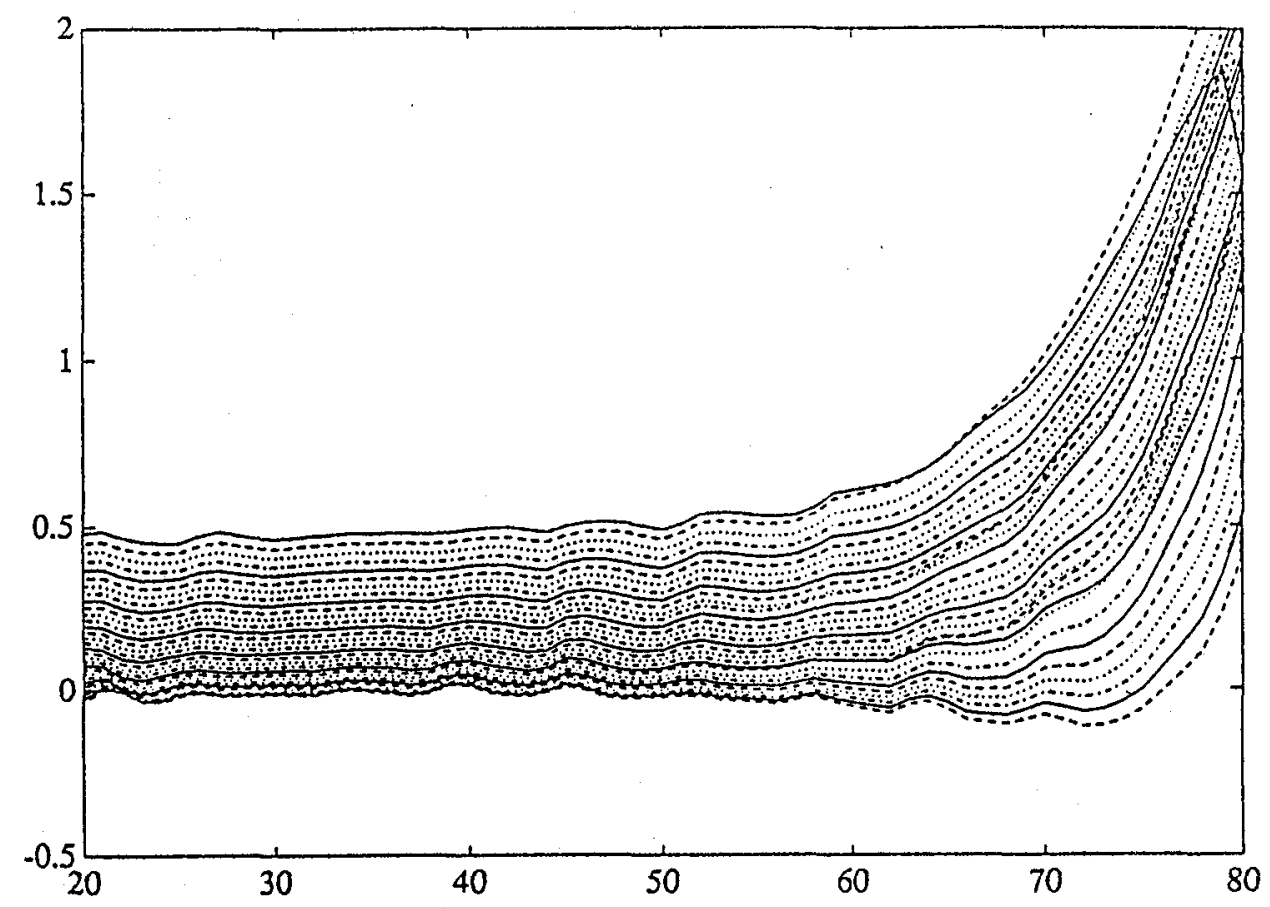

Figure 5. Functions $\alpha(k, t)$ from $t=0.4$ to $t=1$ computed from (4.4). $t$ increases downward. Computation is done on a $256^{2}$ grid.

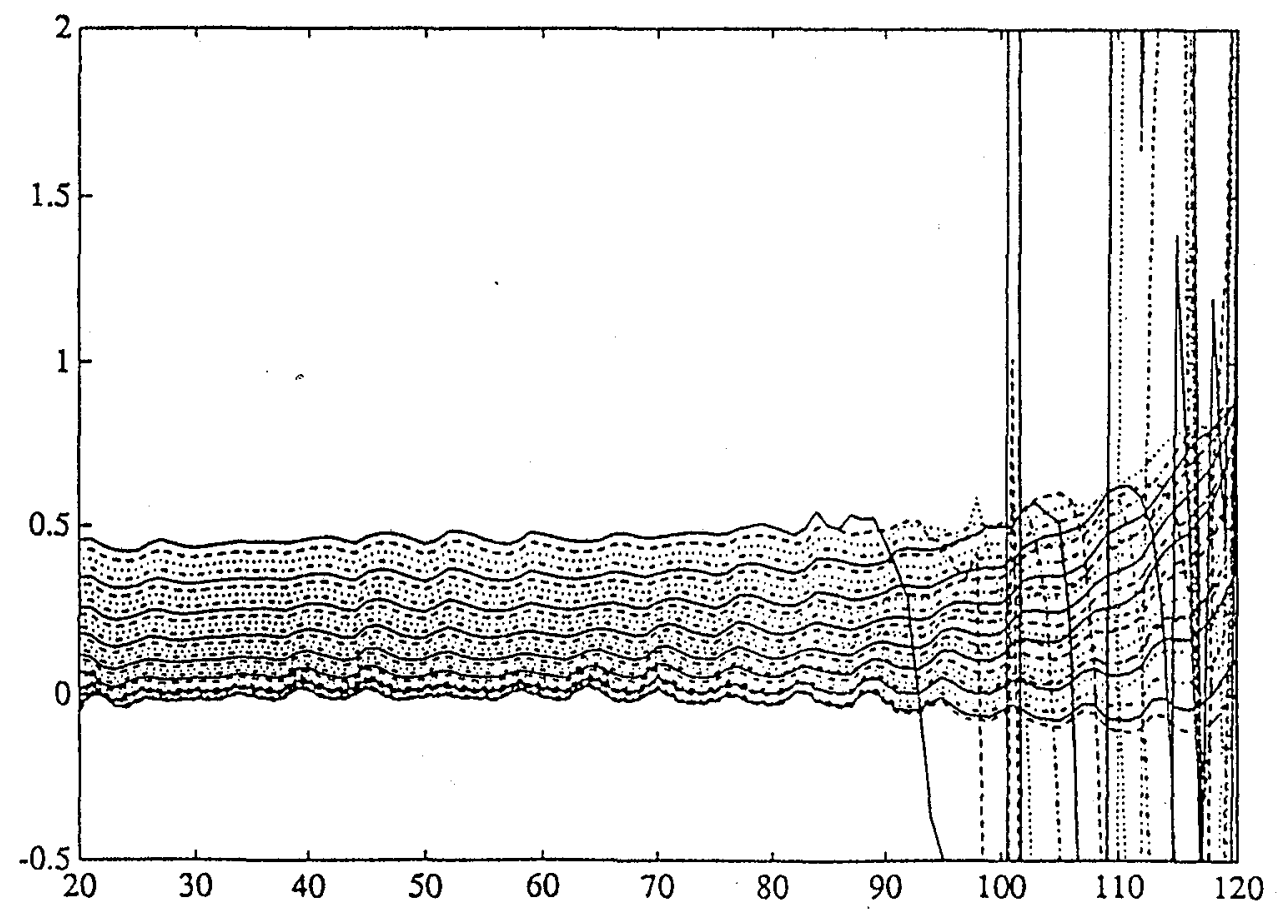

Figure 6. Functions $\alpha(k, t)$ from $t=0.4$ to $t=1$ computed from (4.4). $t$ increases downward. Computation is done on a $400^{2}$ grid. 


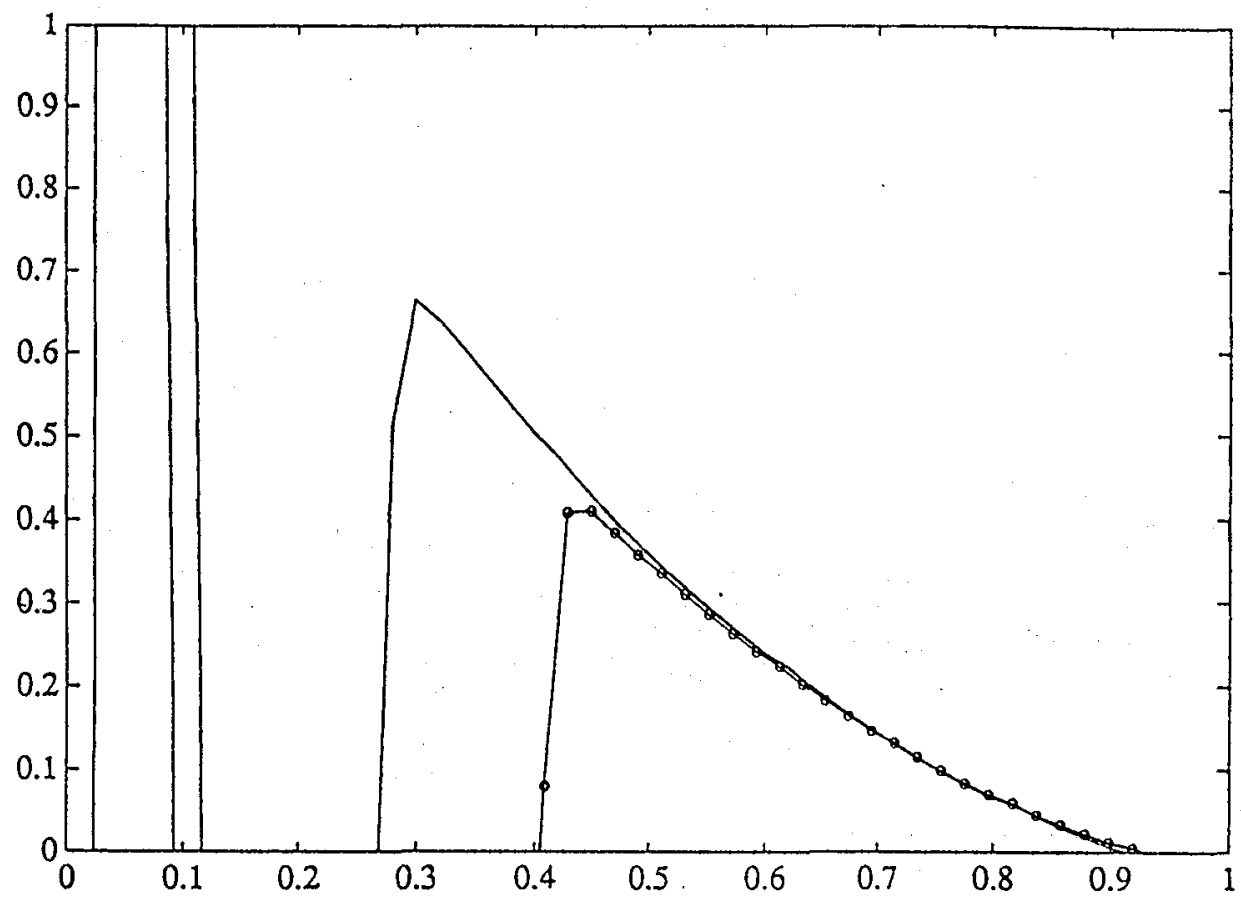

Figure 7. The analyticity strip width $\bar{\alpha}(t)$ computed on different grids. The line with circles is computed on $400^{2}$ grid. The other line is computed on $256^{2}$ grid.

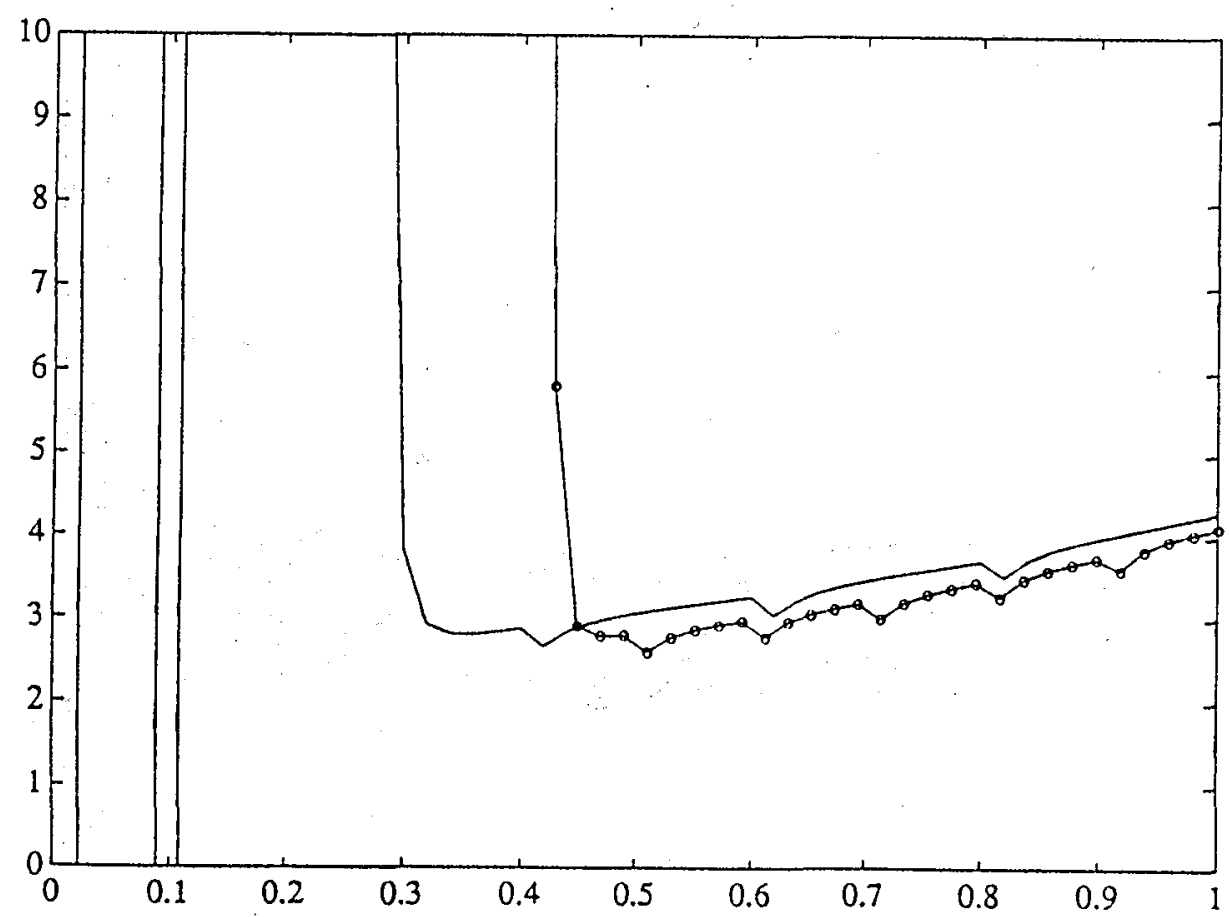

Figure 8. $\bar{\beta}(t)$ computed on different grids. The line with circles is computed on $400^{2}$ grid. The other line is computed on $256^{2}$ grid. 


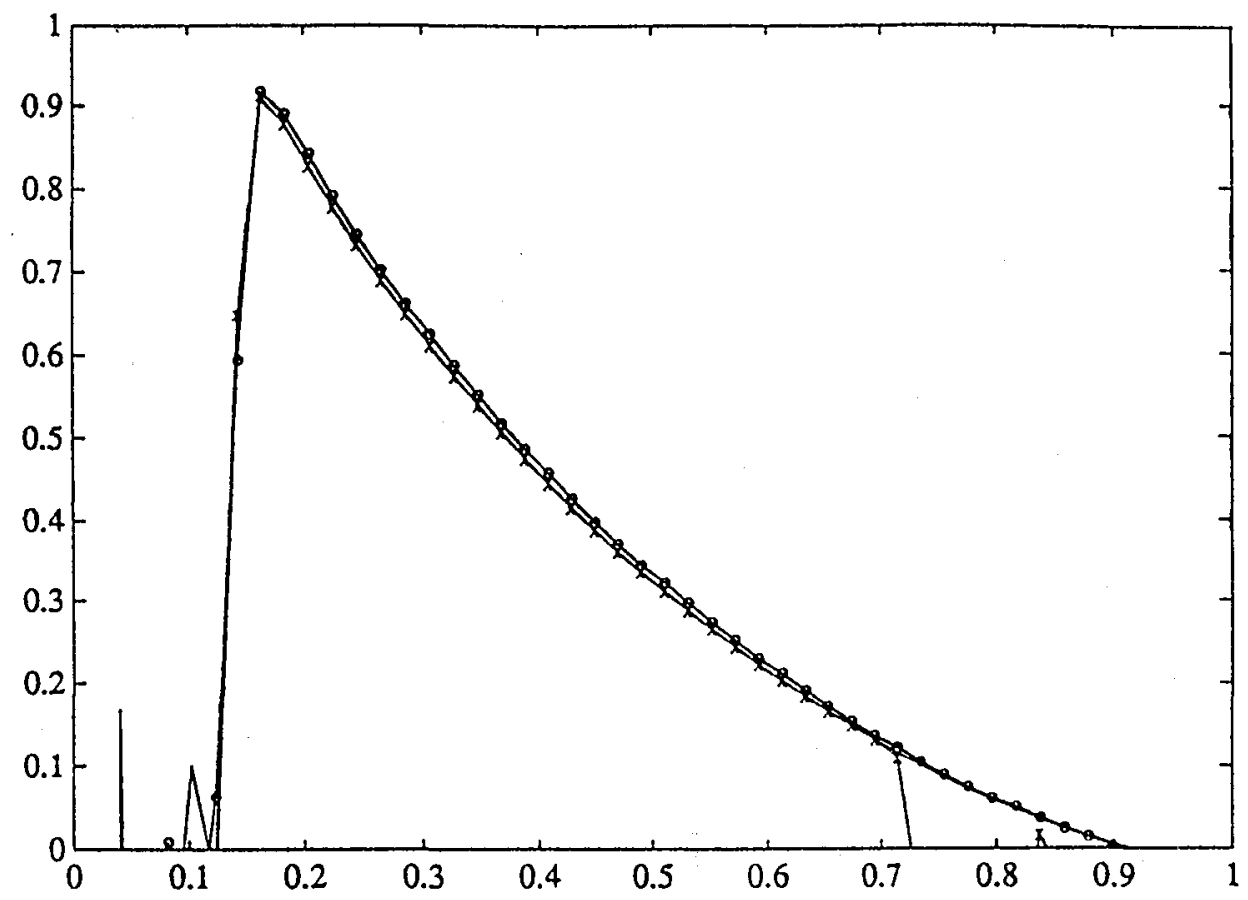

Figure 9. The analyticity strip width $\bar{\alpha}(t)$ computed on a $256^{2}$ grid with different orders of filter. The line with circles is computed with $m_{f}=5$. The line with " $x$ " is computed with $m_{f}=15$. The other line is computed with $m_{f}=10$.

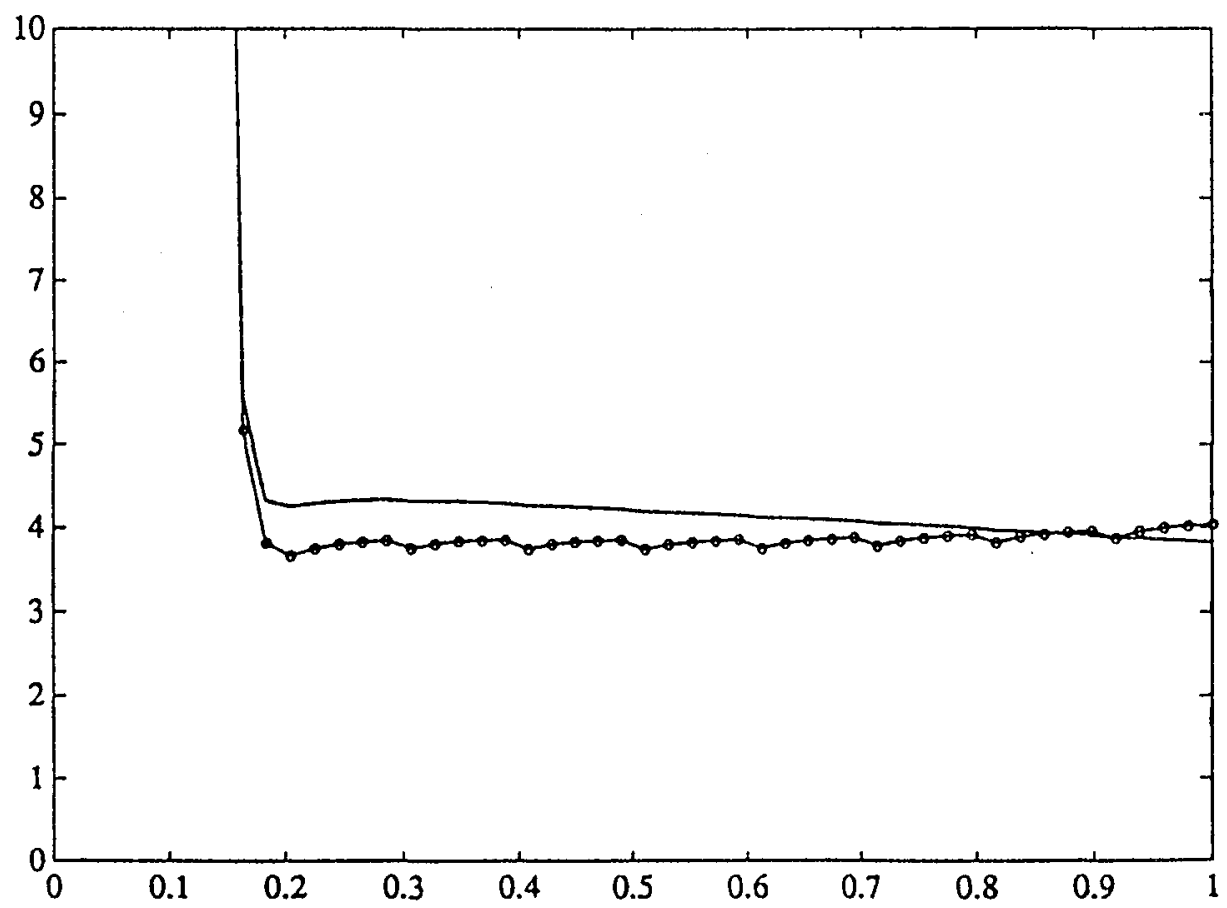

Figure 10. $\bar{\beta}(t)$ computed on a $256^{2}$ grid with different orders of filter. The line with circles is computed with $m_{f}=5$. The other line is computed with $m_{f}=10$. The result for $m_{f}=15$ fluctuates and is not shown here. 


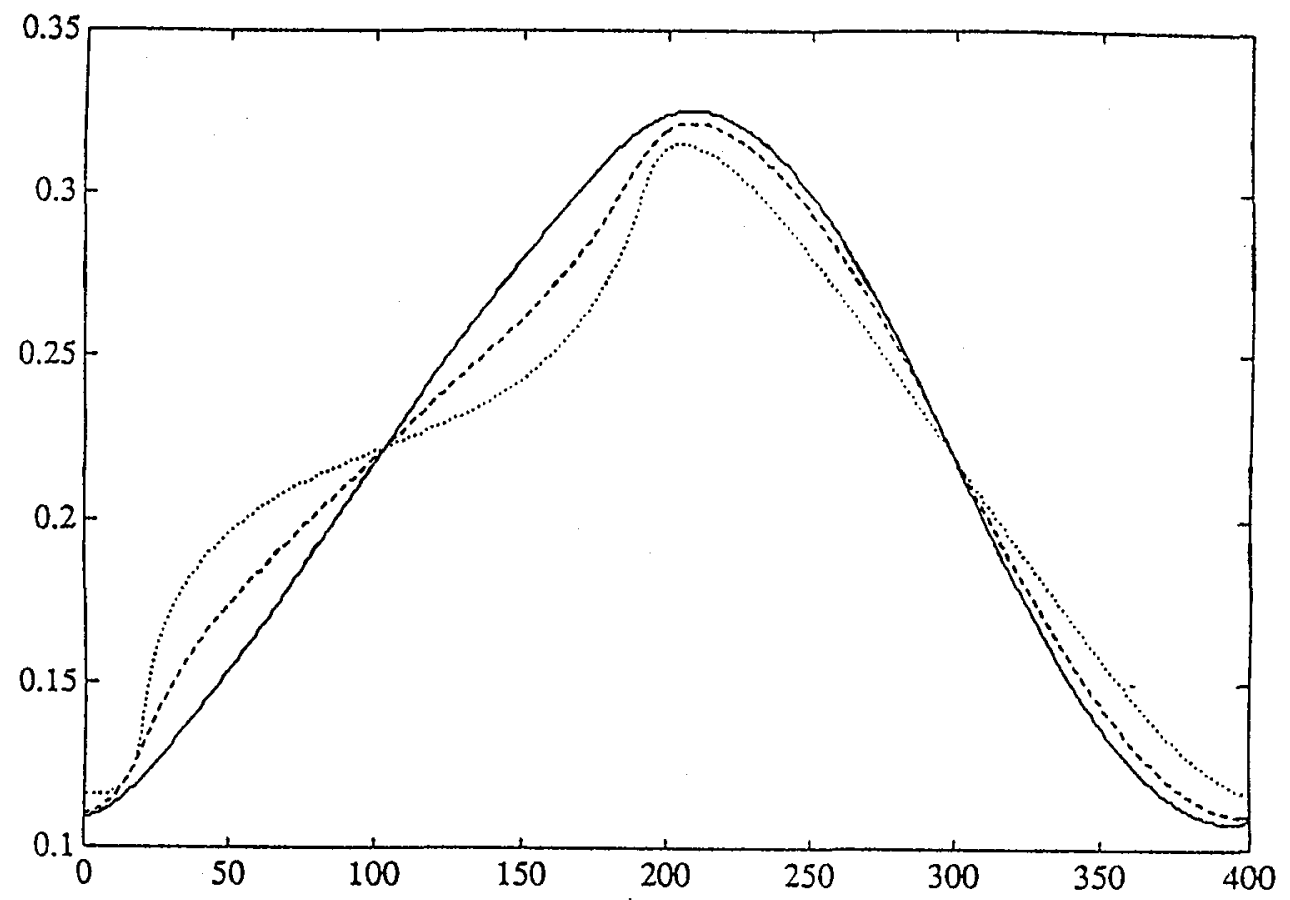

Figure 11. The profile of $u$ at $y=\frac{2 \pi}{3}$ at $t=0.3,0.6$ and 0.9 respectively. The line is for $t=0.3$. The dashed line is for $t=0.6$. The dotted line is for $t=0.9$.

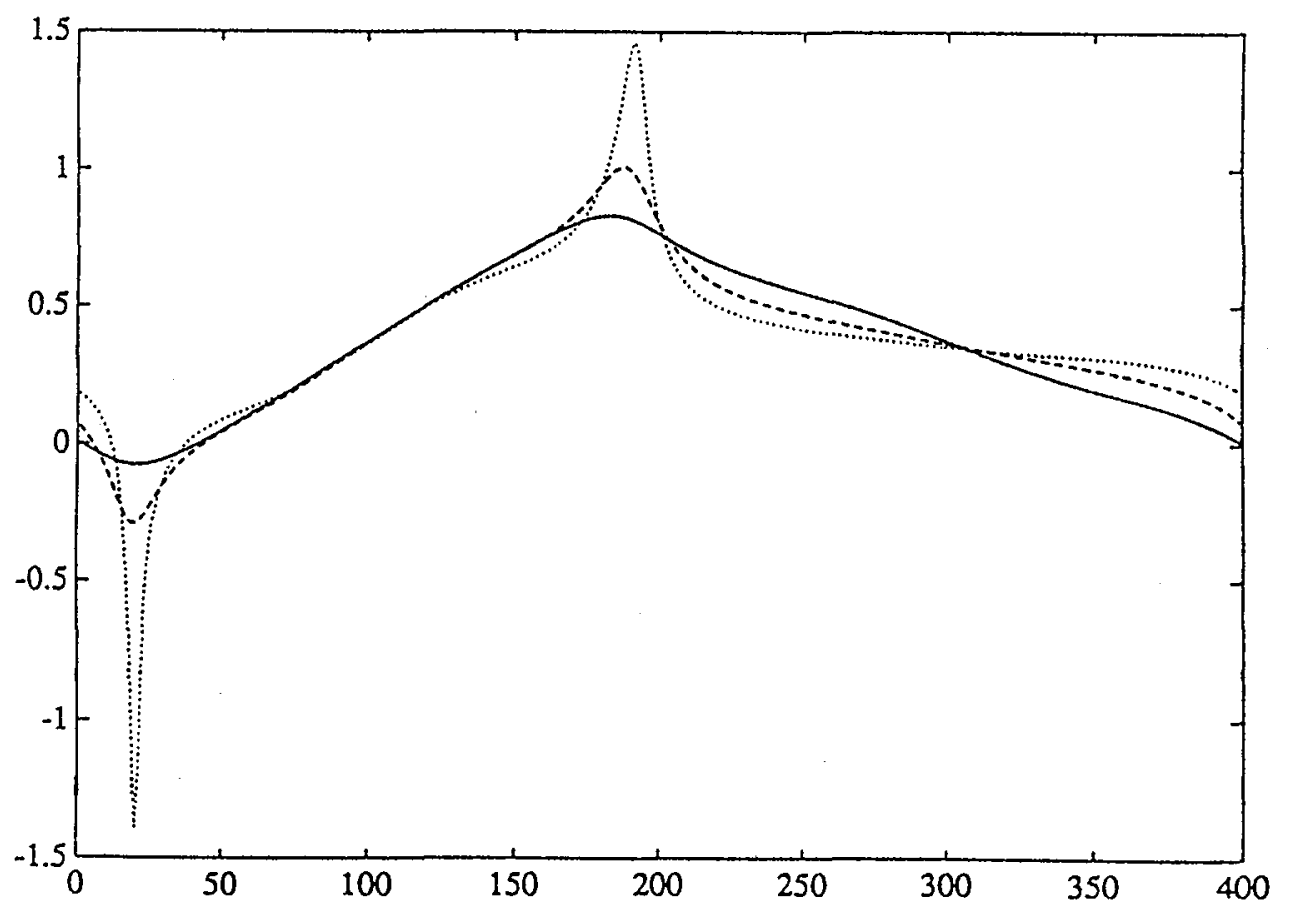

Figure 12. The profile of $\omega$ at $y=\frac{2 \pi}{3}$ at $t=0.3,0.6$ and 0.9 respectively. The line is for $t=0.3$. The dashed line is for $t=0.6$. The dotted line is for $t=0.9$. 



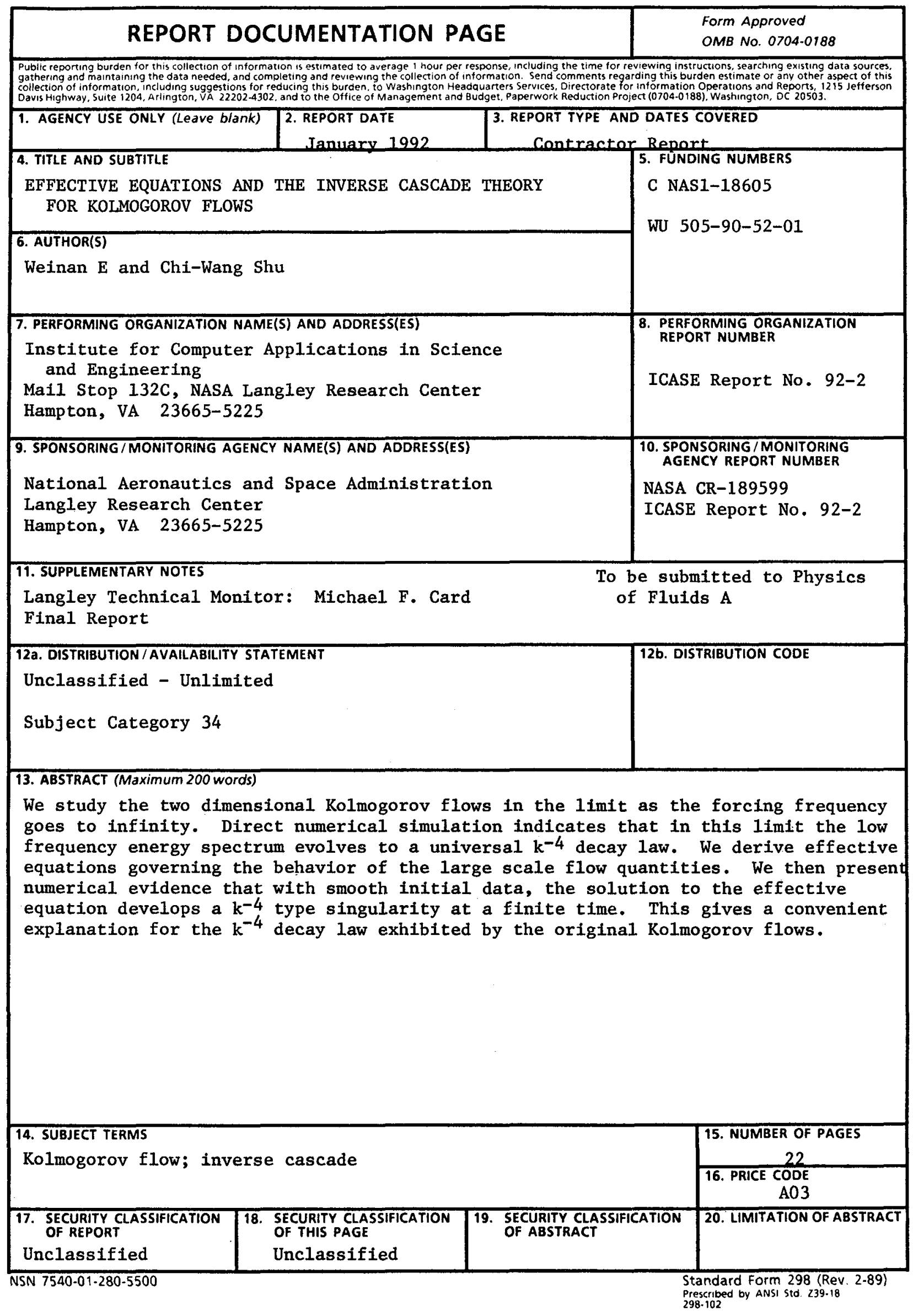





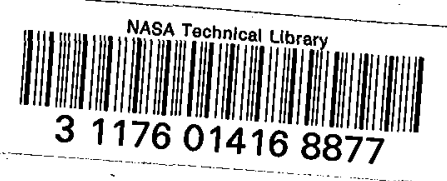

